\title{
Impact of a novel ultrasound microvascular imaging and elastography on prostate cancer classification
}

\author{
Ting-Ting Shen ${ }^{1}$, Jun-Li Xue ${ }^{2}$ \\ ${ }^{1}$ Department of Ultrasound, Shanghai Ji Ai Genetics \& IVF Institute, Shanghai 200011, China; ${ }^{2}$ Department of Oncology, Shanghai Oriental \\ Hospital, Shanghai 200120, China \\ Contributions: (I) Conception and design: TT Shen; (II) Administrative support: JL Xue; (III) Provision of study materials or patients: TT Shen; \\ (IV) Collection and assembly of data: TT Shen; (V) Data analysis and interpretation: All authors; (VI) Manuscript writing: All authors; (VII) Final \\ approval of manuscript: All authors. \\ Correspondence to: Jun-Li Xue. No.1800, Yuntai Road, Pudong, Shanghai, China. Email: 1310666xuejunli@tongji.edu.cn.
}

\begin{abstract}
Background: This study was aimed to compare the sensitivity of transrectal ultrasound (TRUS) guided systematic biopsy (TRUS-SB), superb microvascular imaging guided targeted biopsy (SMI-TB), and elastography ultrasound guided targeted biopsy (EU-TB) in detecting prostate cancer (PCa).

Methods: One hundred and eighty-four patients with suspicious PCa were randomly divided into two groups, SMI-TB group (n=92) and EU-TB group $(\mathrm{n}=92)$. All the patients received a 2-core SMI-TB or a 2-core EU-TB before receiving a 12-core TRU-SB depending on the group. The 2-core SMI-TB was conducted at the most increased vascularity areas rated by a radiologist on a semi-quantitative criterion. The 2-core EU-TB was performed at the dark blue areas that identified as suspicious areas. The PCa detection rate was compared among TRUS-SB, SMI-TB, and EU-TB in the total population and in two groups.

Results: PCa was detected in 65 out of 184 patients. The SMI-TB group patients received 1,272 cores in total with a positive rate of $23.81 \%$ (40/168) for SMI-TB cores and 4.17\% $(46 / 1,104)$ for TRUS-SB cores. The EU-TB group patients received a total of 1,262 cores with a positive rate of $31.01 \%(49 / 158)$ for EUTB cores and 5.34\% (59/1,104) for TRUS-SB cores. Targeted cores $(27.30 \%, 89 / 326)$ achieved significantly higher sensitivity in PCa detection than systematic cores $(4.76 \%, 105 / 2,208)$ (SMI-TB vs. TRUS-SB: OR 7.188, $\mathrm{P}<0.001$; EU-TB vs. TRUS-SB: OR 7.962, $\mathrm{P}<0.001)$.

Conclusions: Superb microvascular imaging and elastography ultrasound guided targeted biopsy may result in higher prostate cancer detection rate as adjunct to gray-scale ultrasound guided systematic biopsy. However, targeted biopsy alone is not sufficient to detect PCa, compared with systematic biopsy.
\end{abstract}

Keywords: Biopsy, ultrasonography, prostatic neoplasms, prostate volume

Submitted Aug 17, 2019. Accepted for publication Nov 05, 2019.

doi: 10.21037/tau.2019.11.15

View this article at: http://dx.doi.org/10.21037/tau.2019.11.15

\section{Introduction}

Prostate cancer (PCa) rarely causes symptoms unless it is advanced. Thus, suspicion of PCa resulting in a recommendation for prostatic biopsy is most often raised by abnormalities found on digital rectal examination (DRE) or by elevated serum prostate-specific antigen (PSA). Transrectal ultrasound (TRUS) guided systematic biopsy (TRUS-SB) is the most commonly used method when there is suspicion of PCa. However, the question of how often a prostate biopsy will turn out to be false-negative is of clinical as well as statistical importance. Daneshgari et al. reported a $25 \%$ chance of missing a PCa using systematic biopsy after examining 112 patients and in which 91 with PCa (1). Rabbani et al. came to a similar finding that $23 \%$ of the 118 patients were failed to identify PCa using the same methodology (2). Thus, various biopsy strategies have been devised to increase the diagnostic yield of prostate 


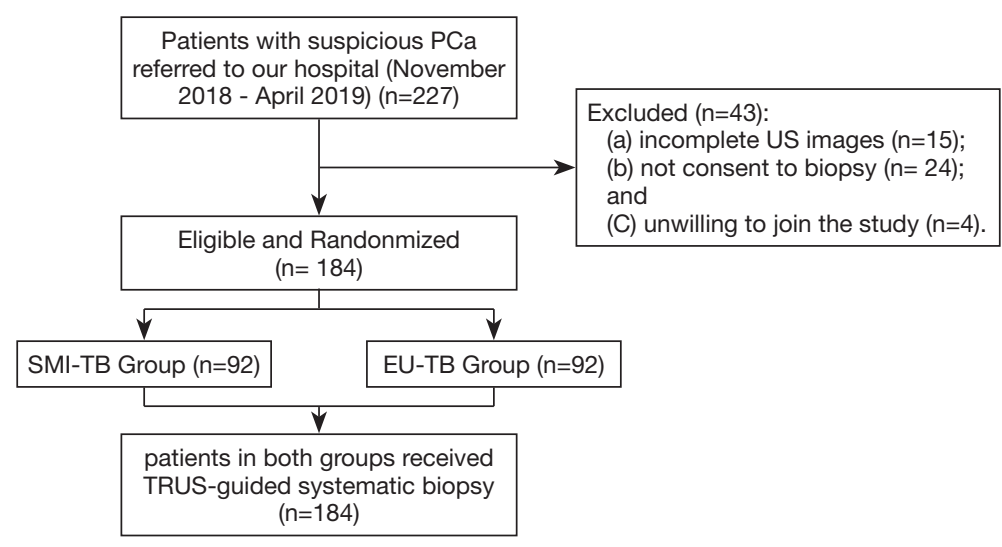

Figure 1 Patient selection flowchart. PCa, prostate cancer; SMI-TB, superb microvascular imaging guided targeted biopsy; EU-TB, elastography ultrasound guided targeted biopsy; TRUS, transrectal ultrasound.

biopsy. Magnetic-resonance imaging (MRI)-guided biopsy is applied in clinical practice. However, MRI-guided biopsy is cost-consuming and time-consuming, which does not fit all patients, especially those with implant, pacemaker, or claustrophobia (3). Color Doppler ultrasonography (CDUS) is one of the tools to increase PCa detection rate (4), owing to the relationship between angiogenesis and tumor growth (5). CDUS interprets the blood velocity and direction by the different frequency shift of US waves and removing nonvascular artifacts by wall filtering (6). However, very low speed vessels might also be dropped if the wall filter setting is too high. That is to say CDUS is poor at microvascular with identifying lower blood flow speed. That might cause a decreased sensitivity in distinguishing less advanced PCa. Superb microvascular imaging (SMI) is considered the next generation of CDUS. It shows the ability of demonstrating very low blood flow signals with less artifacts, applying a multidimensional filter (7). That might give a hint that SMI can provide more detailed vascular signals in correlation with PCa. Researchers came up with a significant positive link between microvascularity and PCa back to 1993 (8). More experiment results demonstrated the feasibility of adding the blood flow information in predicting potential malignancy $(9,10)$.

Besides the interpretation of vascularity, tissue stiffness is also an important factor in predicting malignancy. Previous research tried to figure out the tissue stiffness between benign and malignant lesions using strain elastography ultrasound (EU) $(11,12)$. A better understanding of tissue properties and their distribution in the prostate might increase the accuracy of targeting for tissue acquisition and eventually improve the PCa detection.
However, limited attention has been paid to the application of SMI guided targeted biopsy (SMI-TB) and elastography ultrasound-guided targeted biopsy (EUTB) in detecting PCa. To our best knowledge, no study of distinguishing $\mathrm{PCa}$ by comparing these 3 methods (TRUS-SB, SMI-TB and EU-TB) has been documented. Consequently, the present study was aimed to evaluate which property can better differentiate PCa tissue from normal tissue, and we prospectively evaluated SMI and $\mathrm{EU}$ as guides for TB to evaluate which is more useful in increasing PCa detection when performed before a conventional TRUS-guided systematic biopsy.

\section{Methods}

\section{Patients}

This prospective study was approved by our Ethic Committee (No.056). All enrolled patients signed informed consent forms. From November 2018 to April 2019, 227 patients with suspicious PCa were referred to our hospital. The inclusion criteria of patients was: (I) with a PSA level between $2.5-4.0 \mathrm{ng} / \mathrm{mL}$ and positive DRE; (II) a PSA level above $4.0 \mathrm{ng} / \mathrm{ML}$ with/without positive DRE findings. The exclusion criteria were: (I) patients with no full set of US images; (II) patients with no or incomplete biopsy results; (III) patients refused to join the study. Among these 227 patients, 15 patients did not have complete US images, 24 were not consent to biopsy and 4 patients were unwilling to join the study. Finally, a total of 184 patients were included in this study, being randomly divided into 2 groups, SMITB group (n=92) and EU-TB group (n=92) (Figure 1). 


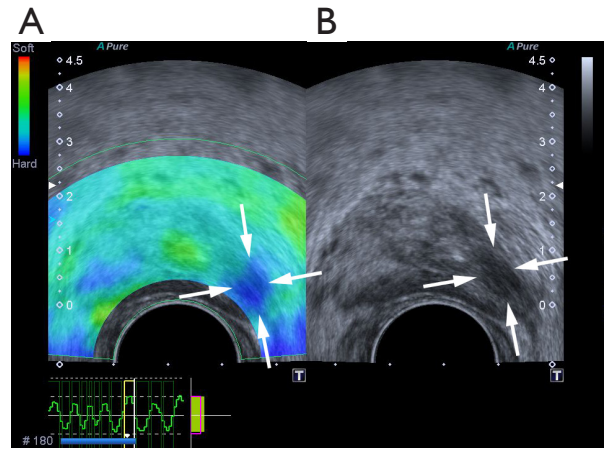

Figure 2 A 76-year-old man with a PSA level of $14.80 \mathrm{ng} / \mathrm{mL}$. (A) EU image reveals a stiffer area (dark blue, arrows) on the left side of the prostate; (B) gray-scale image showing a hypoechoic lesion in the left gland (arrows). Both EU-TB and TRUS-SB confirmed it was prostate cancer. PSA, prostate-specific antigen; EU-TB, elastography ultrasound guided targeted biopsy; TRUS$\mathrm{SB}$, transrectal ultrasound guided systematic biopsy.

\section{Conventional ultrasonography protocol and biopsy}

All patients, in the left lateral position, received TRUS examination by a skilled radiologist with 10 years of experience in prostate imaging. The TRUS exam was performed using the Aplio 500 (Toshiba Medical System Corporation, Tokyo, Japan) with a convex transducer (5-14 MHz). The other radiologist with 15 years' experience in prostate biopsy performed a 12-core TRUS$\mathrm{SB}$, using an 18-gauge needle and automatic biopsy device (Biopty; Bard, Covington, GA) to perform biopsy cores. The 12 cores were taken bilaterally from the base, midzone, and apex of the prostate.

\section{Elastography ultrasound protocol and biopsy}

Patients in US-TB group received US-TB before the systematic biopsy. The same radiologist conducted the elastography exam by repeating the movement of a transrectal probe from above down on the prostate tissue until he obtained stable and reproducible images. The three colors on the image referred to the different degree of the tissue stiffness. A relatively hard tissue (Figure 2) presented as dark blue, while a relatively soft tissue presented as red on the image. Green refers to an average hardness. Pursuant to Konig's criteria, reproducible dark blue areas were identified as suspicious areas (13). Two cores were taken at the most suspicious areas with the same 18G biopsy gun.
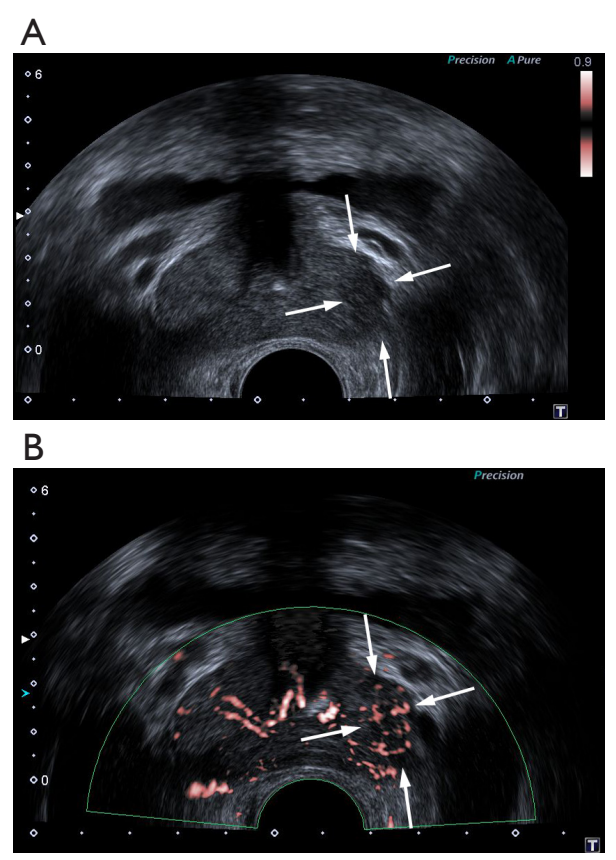

Figure 3 A 68-year-old man with a PSA level of $13.75 \mathrm{ng} / \mathrm{mL}$. (A) Gray-scale image showing a hypoechoic lesion in the left gland (arrows); (B) SMI image showing enrich blood flow signals (arrows). Both SMI-TB and TRUS-SB confirmed it was prostate cancer. SMI-TB, superb microvascular imaging guided targeted biopsy; TRUS-SB, transrectal ultrasound guided systematic biopsy.

\section{Superb microvascular imaging protocol and biopsy}

The same radiologist performed the examination on the same machine with a $7 \mathrm{MHz}$ probe. The velocity scale of SMI was adjusted to $<20 \mathrm{~mm} / \mathrm{s}$. To decrease the influence of probe pressure on vessel flow, the radiologist applied the lowest pressure to the prostate gland. The radiologist detected areas of increased blood flow signals (Figure 3) and rated vascularity on the semi-quantitative criteria. The radiologist who conducted the targeted biopsy then performed 2 cores at the suspicious areas where the most vascularity was found.

\section{Biopsy technique and pathologic analysis}

All biopsy cores were analyzed by the same pathologist who was blind to the US findings. The biopsy specimens were placed with a $10 \%$ formaldehyde solution in different tubes and labelled accordingly. Each core was labeled as TRUS$\mathrm{SB}$, or SMI-TB, or EU-TB. 
Table 1 baseline features of all patients

\begin{tabular}{lccc}
\hline & Malignant $(n=65)$ & Benign $(n=119)$ & $P$ \\
\hline Age (years) & $70.91 \pm 7.82$ & $70.13 \pm 7.39$ & 0.884 \\
PSA $(\mu \mathrm{g} / \mathrm{L})$ & $15.74 \pm 4.94$ & $9.82 \pm 3.54$ & 0.465 \\
Prostate volume $(\mathrm{mL})$ & $36.58 \pm 15.32$ & $43.92 \pm 20.21$ & 0.344 \\
Gleason score & $7.05 \pm 0.86$ & $\mathrm{~N} / \mathrm{A}$ & \\
\hline
\end{tabular}

PSA, prostate-specific antigen.

\section{Statistical analysis}

Continuous quantities were presented in median with interquartile range and analyzed using Student's $t$ test or the Mann-Whitney test. An odds ratio (OR) was utilized to compare the detection rate by SMI-TB/EU-TB versus TRUS-SB. The PCa detection rate by different methods were compared using the Wilcoxon's rank sum test. $\mathrm{P}<0.05$ was considered as statistically significant. SPSS (Version 20.0) was performed for statistical analysis.

\section{Results}

\section{Baseline characteristics}

Demographic characteristics of the 184 men included in this study are summarized in Table 1. The median age, PSA levels, and prostate volume were $70.41 \pm 7.53$ years, $11.93 \pm 5.00 \mathrm{ng} / \mathrm{mL}$, and $41.33 \pm 18.92 \mathrm{~mL}$, respectively. Among them, PCa was detected in 65/184 (35.33\%) patients in this study. The remaining 119 patients were with benign prostatic hyperplasia $(n=90)$ and acute prostatitis $(n=29)$. The mean PSA of PCa patients was $15.74 \pm 4.94 \mathrm{ng} / \mathrm{mL}$ higher than that of benign patients $(9.82 \pm 3.54 \mathrm{ng} / \mathrm{mL})$. However, the difference was not statistically significant $(\mathrm{P}=0.465)$ (Table 1). About 32.31\% (21/65) of PCa patients shown hypoechoic lesions in the peripheral zone (PZ).

\section{"Per patient" analysis}

TRUS showed 163 abnormal lesions in 163 patients. No sonographic abnormality was seen in 21 patients. Among these, $109(66.87 \%)$ were identified in the $\mathrm{PZ}$ and 54 $(33.13 \%)$ were identified in the transition zone (TZ). The overall PCa detection rate was $35.33 \%(65 / 184)$. The true positive rate of $\mathrm{PCa}$ found in the $\mathrm{TZ}(66.15 \%, 43 / 65)$ was higher than that of in the PZ $(33.85 \%, 22 / 65)$. The PCa detection rate was $15.38 \%(10 / 65), 42.57 \%(43 / 101)$, and $92.31 \%(12 / 13)$ in patients with a PSA level of 4-10, 10-20, and $>20 \mu \mathrm{g} / \mathrm{L}$, respectively. The PCa detection rate was $31.52 \%(29 / 92)$ and $39.13 \%(36 / 92)$ in the SMI-TB group and EU-TB group, respectively.

\section{"Per core" analysis}

Patients in the SMI-TB group and EU-TB group received a total of 1,272 (SMI-TB: 168; TRUS-SB: 1,104) and 1,262 (EU-TB: 158; TRUS-SB: 1,104) and biopsy cores, respectively. 40 of 168 (23.81\%) SMI-TB cores were positive for PCa, while 46 of 1,104 (4.17\%) TRUS-SB cores were positive for PCa in the SMI-TB group. 49 of 158 (31.01\%) EU-TB cores were positive for PCa, while 59 of 1,104 (5.34\%) TRUS-SB cores were positive for PCa in the EU-TB group. In total, targeted cores $(27.30 \%$, 89/326) were significantly more likely than systematic cores $(4.76 \%, 105 / 2,208)$ to distinguish PCa (SMI-TB vs. TRUSSB: OR=7.188, $\mathrm{P}<0.001$; EU-TB vs. TRUS-SB: OR=7.962, $\mathrm{P}<0.001)$.

\section{Discussion}

With the advanced prostate imaging modes, targeted biopsy under imaging guidance may provide a practical solution. SMI and EU are two novel ultrasound techniques with the advantage of being non-intuitive, non-radiative, and cost-efficient. In this study, we evaluated the sensitivity of PCa detection by SMI-TB and EU-TB, comparing with conventional TRUS-SB. Our results demonstrated that the sensitivity of PCa recognition by targeted biopsy was significantly higher than that by TRUS-SB. Four additional PCa patients were identified by using SMI-TB adjunct to TRUS-SB and 3 additional positive cases were identified by using EU-TB adjunct to TRUS-SB.

The deposition of collagen increases in the stroma around the prostate lesion and decreases in the acinar region $(14,15)$. That may result in easier identification of PCa since neoplastic prostate tissues are usually stiffer than the noncancerous tissues (16). Pallwein et al. reported an $87 \%$ of sensitivity applying sonoelastography guided targeted biopsy and demonstrated a good correlation with the 10-core TRUS SB (17). The researchers suggested that sonoelastography might offer a new method for PCa detection. Aigner et al. evaluated 94 patients who underwent both EU-TB and TRUS-SB and came up with a promising finding that targeted cores detected PCa 4.7 times higher than systematic cores (18). Our results were comparable with those of the published results. In the present study, 
163 of 184 patients with suspicious TRUS findings, 65 were diagnosed with PCa (SMI-TB group: $\mathrm{n}=29$; and EU-TB group: $\mathrm{n}=36$ ). EU-TB identified 26 out of $36 \mathrm{PCa}$ patients with a sensitivity rate of $31.01 \%$ (49/158) of targeted cores. In comparison, 59 out of 1,104 systematic cores determined positive cases. The EU-TB cores classified PCa 5.81 times more often than TRUS-SB cores.

Increased micro-vascularity such as tumor-associated angiogenesis and loss of glandular architecture are commonly observed in the morphological changes of malignant prostate tissues (8). Tang et al. demonstrated a positive correlation between prostate cancer-associated vascularity and PCa grade (19). Their study detected abnormal vascular information in $90.7 \%$ of PCa. Sen et al. reported $88.2 \%$ of $\mathrm{PCa}$ but $33.3 \%$ of benign lesions shown increased vessels (20). Our findings were comparable with that of previous publications. In the present study, SMI detected vascular signals in 25 of $29 \mathrm{PCa}(86.21 \%)$ and 20 of $63(31.75 \%)$ benign prostate lesions $(\mathrm{P}<0.001)$. In addition, SMI-TB offered a good sensitivity in PCa detection in terms of per core analysis. SMI-TB cores $(23.81 \%, 40 / 168)$ were more sensitive in PCa detection than TRUS-SB cores $(4.34 \%, 46 / 1,058)(\mathrm{OR}=7.188, \mathrm{P}<0.001)$. There is no published article on the role of SMI in PCa recognition. However, we found out that our results were similar to those investigated contrast-enhanced ultrasound (CEUS) targeted biopsy (CEUS-TB) in PCa detection. Previous study has demonstrated the good consistency between SMI and CEUS in depicting vascularity information (21). Colleselli et al. prospectively evaluated 345 men with PCa using a combined method of CEUS-TB and TRUS-SB (22). They demonstrated a significantly higher detection rate by CEUS-TB $(88.1 \%)$ in small prostate glands $(<30 \mathrm{~mL})$ compared with that by TRUS-SB (69.0\%). However, some studies debated that the improvement in PCa detection was not ideal (23). In our study, SMI-TB missed 20.69\% (6/29) PCa while TRUS-SB missed $13.79 \%$ (4/29). That might due to the finding that SMI may be more sensitive for detection of clinically significant lesions. The average Gleason score in SMI-TB group was 7.38 \pm 0.90 , lower than and the average Gleason score of PCa detected by SMI-TB was $7.61 \pm 0.84$. Doppler flow associated with higher Gleason scores has been confirmed by prior researches $(24,25)$.

Recent studies suggested that increasing number of cores on systematic biopsy can improve PCa detection (26). However, biopsy is invasive which may cause serious complications in certain cases (27). In addition, increasing of the biopsy cores on systematic biopsy correlates with additional cost. Previous literatures argued that 20-core biopsy had no significant advantage over 12-core biopsy (28). The present study suggested an increased sensitivity with a decreased number of cores, applying either SMI-TB or EU-TB. We believe that targeted biopsy may provide great assist to detect PCa with fewer numbers of biopsy cores. That would eventually lower the morbidity associated with biopsy in patients with suspicious prostate lesions.

Our study has certain limitations. First, it was a prospective study with a small number of participants. Further studies with larger study sample is needed to validate our results. Secondly, all the ultrasound images were performed by the same operator in this study. Ultrasound examination is operator-dependent and readerdependent. Therefore, further studies are needed to control of interobserver differences in the assessment of US images.

\section{Conclusions}

Our results suggest that vascular features assessed by superb microvascular imaging (SMI) and tissue stiffness assessed by elastography ultrasound (EU) may result in higher prostate cancer $(\mathrm{PCa})$ detection rate as adjunct to grayscale ultrasound. Targeted biopsy alone is not competent to detect PCa.

\section{Acknowledgments}

Funding: This study was funded by the Outstanding Clinical Discipline Project of Shanghai Pudong (No. PWYgy2018-02).

\section{Footnote}

Conflicts of Interest: The authors have no conflicts of interest to declare.

Ethical Statement: The authors are accountable for all aspects of the work in ensuring that questions related to the accuracy or integrity of any part of the work are appropriately investigated and resolved. This prospective study was approved by Shanghai Oriental Hospital Ethic Committee (No.056). All enrolled patients signed informed consent forms. 


\section{References}

1. Daneshgari F, Taylor GD, Miller GJ, et al. Computer simulation of the probability of detecting low volume carcinoma of the prostate with six random systematic core biopsies. Urology 1995;45:604-09.

2. Rabbani F, Stroumbakis N, Kava BR, et al. Incidence and clinical significance of false-negative sextant prostate biopsies. J Urol 1998;159:1247-50.

3. Yunkai Z, Yaqing C, Jun J, et al. Comparison of contrastenhanced ultrasound targeted biopsy versus standard systematic biopsy for clinically significant prostate cancer detection: result of a prospective cohort study with 1024 patients. World J Urol 2019;37:805-11.

4. Borre M, Offersen BV, Nerstrøm B, et al. Microvessel density predicts survival in prostate cancer patients subjected to watchful waiting. Br J Cancer 1998;78:940-4.

5. Folkman J. Angiogenesis in cancer, vascular, rheumatoid and other disease. Nat Med 1995;1:27-31.

6. Szabo TL. Doppler Modes. In: Diagnostic Ultrasound Imaging: Inside Out. Elsevier, 2014;431-500.

7. Zhu YC, Zhang Y, Deng SH. A prospective study to compare superb microvascular imaging with grayscale ultrasound and color Doppler flow imaging of vascular distribution and morphology in thyroid nodules. Med Sci Monit 2018;24:9223-31.

8. Bigler SA, Deering RE, Brawer MK. Comparison of microscopic vascularity in benign and malignant prostate tissue. Hum Pathol 1993;24:220-6.

9. Lissbrant IF, Stattin P, Damber JE, et al. Vascular density is a predictor of cancer-specific survival in prostatic carcinoma. Prostate 1997;33:38-45.

10. Khanduri S, Katyal G, Goyal A, et al. Evaluation of Prostatic Lesions by Transrectal Ultrasound, Color Doppler, and the Histopathological Correlation. Cureus 2017;9:e1422.

11. Zhai L, Madden J, Foo WC, et al. Characterizing stiffness of human prostates using acoustic radiation force. Ultrason Imaging 2010;32:201-3.

12. Wang Y, Yao B, Li H, et al. Assessment of tumor stiffness with shear wave elastography in a human prostate cancer xenograft implantation model. J Ultrasound Med 2017;36:955-63.

13. König K, Scheipers U, Pesavento A, et al. Initial experiences with real-time elastography guided biopsies of the prostate. J Urol 2005;174:115-7.

14. Tuxhorn JA, Ayala GE, Smith MJ, et al. Reactive stroma in human prostate cancer: induction of myofibroblast phenotype and extracellular matrix remodeling. Clin Cancer Res 2002;8:2912-23.

15. Zhang Y, Nojima S, Nakayama H, et al. Characteristics of normal stromal components and their correlation with cancer occurrence in human prostate. Oncol Rep 2003;10:207-11.

16. Krouskop TA, Wheeler TM, Kallel F, et al. Elastic moduli of breast and prostate tissues under compression. Ultrason Imaging 1998;20:260-74.

17. Pallwein L, Mitterberger M, Pinggera G, et al. Sonoelastography of the prostate: comparison with systematic biopsy findings in 492 patients. Eur J Radiol 2008;65:304-10.

18. Aigner F, Pallwein L, Junker D, et al. Value of realtime elastography targeted biopsy for prostate cancer detection in men with prostate specific antigen $1.25 \mathrm{ng} / \mathrm{ml}$ or greater and $4.00 \mathrm{ng} / \mathrm{ml}$ or less. J Urol 2010;184:913-7.

19. Tang J, Li S, Li J, et al. Correlation between prostate cancer grade and vascularity on color doppler imaging: preliminary findings. J Clin Ultrasound 2003;31:61-8.

20. Sen J, Choudhary L, Marwah S, et al. Role of colour Doppler imaging in detecting prostate cancer. Asian J Surg 2008;31:16-9.

21. Zhu YC, Jiang XZ, Bai QK, et al. Evaluating the efficacy of Atorvastatin on patients with carotid plaque by an innovative ultrasonography. J Stroke Cerebrovasc Dis 2019;28:830-7.

22. Colleselli D, Bektic J, Schaefer G, et al. The influence of prostate volume on prostate cancer detection using a combined approach of contrast-enhanced ultrasonography-targeted and systematic grey-scale biopsy. BJU Int 2007;100:1264-7.

23. Nelson ED, Slotoroff CB, Gomella LG, et al. Targeted biopsy of the prostate: the impact of color doppler imaging and elastography on prostate cancer detection and gleason score. Urology 2007;70:1136-40.

24. Cornud F, Hamida K, Flam T, et al. Endorectal color Doppler sonography and endorectal MR imaging features of nonpalpable prostate cancer: correlation with radical prostatectomy findings. AJR Am J Roentgenol 2000;175:1161-68.

25. Ismail M, Petersen RO, Alexander AA, et al. Color Doppler imaging in predicting the biologic behavior of prostate cancer: correlation with disease-free survival. Urology 1997;50:906-12.

26. Elabbady AA, Khedr MM. Extended 12-core prostate 
biopsy increases both the detection of prostate cancer and the accuracy of Gleason score. Eur Urol 2006;49:49-53.

27. Schwarzman LS, Abern MR, Garvey DF, et al. The association of previous prostate biopsy related complications and the type of complication with patient compliance with rebiopsy scheme. J Urol 2018;200:1062-7.

28. Irani J, Blanchet P, Salomon L, et al. Is an extended 20core prostate biopsy protocol more efficient than the standard 12-core? A randomized multicenter trial. J Urol 2013;190:77-83.
Cite this article as: Shen TT, Xue JL. Impact of a novel ultrasound microvascular imaging and elastography on prostate cancer classification. Transl Androl Urol 2019;8(6):696-702. doi: $10.21037 /$ tau.2019.11.15 